\title{
JORDAN ALGEBRAS OF DEGREE 1
}

BY K. MCCRIMMON

Communicated by N. Jacobson, April 20, 1964

For finite-dimensional special Jordan algebras, A. A. Albert proved [1] the following

THEOREM. Let $A$ be a Jordan algebra over a field $\Phi$ of characteristic $\neq 2$. If $A$ has an identity element 1 such that every $a \in A$ is of the form $a=\lambda 1+z$ for $\lambda \in \Phi, z$ nilpotent, then $A=\Phi 1+Z$ where $Z$ is a nil subalgebra.

The concepts of inverses and ternary compositions in Jordan algebras were first introduced by $\mathrm{N}$. Jacobson to adapt Albert's argument to the general finite-dimensional case. The following proof shows that the theory of inverses as developed in [2], [3] and subsequently can be used to yield the result directly without any dimensionality restrictions.

Proof. Let $Z=\{z \mid z$ nilpotent $\}$; note that $\lambda 1+z$ is regular if $\lambda \neq 0$ since it has an inverse in $\Phi[z]$, so $Z=\{z \mid z$ singular $\}=\left\{z \mid U_{z}\right.$ singular $\}$, and hence the fundamental formula $U_{U(x) z}=U_{x} U_{z} U_{x}$ shows $U_{x} z \in Z$ for all $x \in A, z \in Z$. We must show $Z$ is a subalgebra; by commutativity it suffices to show $a, b \in Z \Rightarrow \lambda a, a^{2}, a+b \in Z$. Clearly $\lambda a \in Z$, and $a^{2} \in Z$ since $A$ is power-associative. Suppose $a+b \notin Z$; multiplying by a scalar we may assume $a+b=1-4 z$ for $z \in Z$. But $1-4 z$ has a regular square root: if $\lambda_{1}=1, \lambda_{k}=\sum_{1 \leq i \leq k-1} \lambda_{i} \lambda_{k-i}$ then the $\lambda_{k}$ are integers, $w=\sum_{k \geq 1} \lambda_{k} z^{k}$ is a well-defined nilpotent element of $A, w-w^{2}=z$, so $c=1-2 w$ is regular and $c^{2}=1-4 w+4 w^{2}=1-4 z$. Thus $a+b=c^{2}$ $=U_{c} 1$, so $a^{\prime}+b^{\prime}=U_{c^{-1}} a+U_{c^{-1}} b=U_{c^{-1}} U_{c} 1=1$ for $a^{\prime}, b^{\prime} \in Z$, which is a contradiction since $b^{\prime}=1-a^{\prime}$ is regular if $a^{\prime}$ is nilpotent. Hence we must have $a+b \in Z$.

\section{REFERENCES}

1. A. A. Albert, $A$ theory of power associative commutative algebras, Trans. Amer. Math. Soc. 69 (1950), 503-527.

2. N. Jacobson, $A$ theorem on the structure of Jordan algebras, Proc. Nat. Acad. Sci. U.S.A. 42 (1956), 140-147.

3. - A coordinatization theorem for Jordan algebras, Proc. Nat. Acad. Sci. U.S.A. 48 (1962), 1154-1160.

YALE UNIVERSITY 